\title{
Recovery algorithms for high-dimensional rank one tensors
}

\author{
David Krieg \\ Mathematisches Institut, Universität Jena \\ Ernst-Abbe-Platz 2, 07743 Jena, Germany \\ email: david.krieg@uni-jena.de
}

Daniel Rudolf

Institut für Mathematische Stochastik, Universität Göttingen

Goldschmidtstr. 3-5, 37077 Göttingen, Germany

email: daniel.rudolf@uni-goettingen.de

August 9, 2021

\begin{abstract}
We present deterministic algorithms for the uniform recovery of $d$-variate rank one tensors from function values. These tensors are given as product of $d$ univariate functions whose $r$ th weak derivative is bounded by $M$. The recovery problem is known to suffer from the curse of dimensionality for $M \geq 2^{r} r$. For smaller $M$, a randomized algorithm is known which breaks the curse. We construct a deterministic algorithm which is even less costly. In fact, we completely characterize the tractability of this problem by distinguishing three different ranges of the parameter $M$.
\end{abstract}

Keywords: High dimensional approximation, rank one tensors, worst case error, tractability, curse of dimensionality, dispersion.

Classification: Primary: 65Y20; Secondary: 41A15, 41A25, 41A63, 65D99. 


\section{Introduction}

Suppose we know that a $d$-variate function $f$ is the product of $d$ univariate functions with a certain smoothness. How many function values do we need to capture $f$ up to some error $\varepsilon \in(0,1)$ in the uniform norm? This question has been posed and investigated in the work of Bachmayr, Dahmen, DeVore and Grasedyck [2]. The hope is that the structural knowledge about $f$ allows for efficient deterministic approximation schemes in high-dimensional settings. More precisely, it is assumed that $f$ is contained in the class of rank one tensors given by

$$
F_{r, M}^{d}=\left\{\bigotimes_{i=1}^{d} f_{i} \mid f_{i}:[0,1] \rightarrow[-1,1],\left\|f_{i}^{(r)}\right\|_{\infty} \leq M\right\}
$$

for smoothness parameters $r \in \mathbb{N}$ and $M>0$. Here, $f_{i}^{(r)}$ denotes the $r$ th weak derivative of $f_{i}$. In particular, it is assumed that $f_{i}$ is contained in the class $W_{\infty}^{r}([0,1])$ of univariate functions which have $r$ weak derivatives in $L_{\infty}([0,1])$.

It is proven in [8] that for $M \geq 2^{r} r$ ! this problem suffers from the curse of dimensionality: To ensure an error smaller than $\varepsilon$, any deterministic algorithm must use exponentially many function values with respect to the dimension. Even for randomized methods, the curse is present. For $M<2^{r} r$ ! however, a randomized algorithm is constructed which does not require exponentially many function values. We are driven by the question whether the same is possible with a deterministic algorithm. We give an affirmative answer to this question. In fact, we explicitly construct and analyze deterministic algorithms for different ranges of the smoothness parameters. We use the following terminology.

The worst case error of an algorithm $A$ on the class $F_{r, M}^{d}$ is given by

$$
\mathrm{e}(A):=\sup _{f \in F_{r, M}^{d}}\|f-A(f)\|_{\infty}
$$

The number of function values used by $A$ for the input $f$ is $\operatorname{denoted}$ by $\operatorname{cost}(A, f)$. The worst case cost of $A$ is given by

$$
\operatorname{cost}(A):=\sup _{f \in F_{r, M}^{d}} \operatorname{cost}(A, f)
$$

A deterministic algorithm is already constructed in [2]. It achieves the worst 
case error $\varepsilon$ while using at most

$$
C_{r, d} M^{d / r} \varepsilon^{-1 / r}
$$

function values of $f$, see [2, Theorem 5.1]. This number behaves optimally as a function of $\varepsilon$. However, the constant $C_{r, d}$ and hence the number of function values grows super-exponentially with $d$ for any $M>0$ and $r \in \mathbb{N}$. For the algorithm, the following observation of Bachmayr, Dahmen, DeVore and Grasedyck is crucial. If we know some $z^{*} \in[0,1]^{d}$ with $f\left(z^{*}\right) \neq 0$, we can construct a method $I_{m}\left(z^{*}, \cdot\right)$ that uses $m$ function values and satisfies

$$
\left\|I_{m}\left(z^{*}, f\right)-f\right\|_{\infty} \leq \varepsilon
$$

if we choose

$$
m=\left\lfloor C_{r, M} d^{1+1 / r} \varepsilon^{-1 / r}\right\rfloor .
$$

Here, $C_{r, M}$ is a positive constant which only depends on $r$ and $M$. For example, one can choose $C_{r, M}=4 \max \left\{1, C_{1}(r) M\right\}^{1 / r}$ with $C_{1}(r)$ as in [2, Section 2]. Roughly speaking, the knowledge of a non-zero of $f$ allows us to reduce the problem to $d$ univariate approximation problems which can, for example, be treated by the use of polynomial interpolation. With this observation at hand, the authors of [2] use an approximation scheme of the following type:

Algorithm 1. Given $m \in \mathbb{N}$, a finite point set $P \subset[0,1]^{d}$ and a function $f \in F_{r, M}^{d}$, obtain $A_{P, m}(f)$ as follows:

1. For any $x \in P$ check whether $f(x) \neq 0$.

2. If we found some $z^{*} \in P$ with $f\left(z^{*}\right) \neq 0$ then call $I_{m}\left(z^{*}, f\right)$ from (1). If $f_{\mid P}=0$, then return the zero function.

The idea behind this algorithm is to choose $P$ such that whenever $f_{\mid P}=0$, then $\|f\|_{\infty}$ must be small and the zero function is a good approximation of $f$. The authors of [2] use a point set $P$, which contains a finite Halton sequence $H$. They obtain that $f_{\mid P}=0$ implies

$$
\|f\|_{\infty} \leq(2 M)^{d}\left(2^{d} \pi_{d}\right)^{r}|H|^{-r},
$$

where $\pi_{d}$ is the product of the first $d$ primes. To ensure an error bound smaller 
than $\varepsilon$ one needs

$$
|P| \geq|H| \geq\left(2^{d} M^{d} \varepsilon^{-1}\right)^{1 / r}\left(2^{d} \pi_{d}\right)
$$

function evaluations of $f$. However, this number increases super-exponentially with the dimension for all parameters $M$ and $r$.

We also use Algorithm 1 but for smaller point sets $P$. To give a better intuition of the role of the point set, we introduce the notion of detectors. We call a finite point set $P$ in $[0,1]^{d}$ an $\varepsilon$-detector for the class $F_{r, M}^{d}$ if it contains (detects) a non-zero of every function $f \in F_{r, M}^{d}$ with uniform norm greater than $\varepsilon$. If $P$ is an $\varepsilon$-detector for $F_{r, M}^{d}$ and $m$ is chosen as in (2), it is easy to see that Algorithm 1 satisfies

$$
\mathrm{e}\left(A_{P, m}\right) \leq \varepsilon \quad \text { and } \quad \operatorname{cost}\left(A_{P, m}\right) \leq|P|+m
$$

see Lemma 1. We thus need to construct small $\varepsilon$-detectors $P$ for $F_{r, M}^{d}$.

In the range $M \geq 2^{r} r$ ! we know that the problem suffers from the curse of dimensionality such that we cannot expect to find an $\varepsilon$-detector with small cardinality. In that case, we provide a detector for which the cardinality of the point set depends exponentially on $d$. In the range $M<2^{r} r$ ! we give a detector whose cardinality only grows polynomially with $d$. The order of growth is proportional to $\log _{2}\left(\varepsilon^{-1}\right)$. For $M \leq r$ ! the point set can be chosen even smaller. There is a detector whose cardinality grows quadratically with $d$, at worst, regardless of the value of $\varepsilon$. Altogether, we obtain the following:

Theorem 1. For any $r \in \mathbb{N}$ and $M>0$, there are positive constants $c_{i}, i \leq 4$, such that the following holds. For any $d \in \mathbb{N}$ and $\varepsilon \in(0,1)$, there is a finite point set $P \subset[0,1]^{d}$ and a natural number $m$ such that $\mathrm{e}\left(A_{P, m}\right) \leq \varepsilon$ and

$$
\operatorname{cost}\left(A_{P, m}\right) \leq\left\{\begin{array}{lr}
c_{1}^{d} \varepsilon^{-1 / r} & \text { if } M \in(0, \infty), \\
c_{2} \exp \left(c_{3}\left(1+\ln \left(\varepsilon^{-1}\right)\right)(1+\ln d)\right) & \text { if } M \in\left(0,2^{r} r !\right), \\
c_{4} d^{2} \varepsilon^{-1 / r} \ln \left(\varepsilon^{-1 / r}\right) & \text { if } M \in(0, r !]
\end{array}\right.
$$

We always choose $m$ as in (2). The point sets $P$ and the constants $c_{i}$ can be found in Section 2. In each of these ranges we also give a lower bound on the complexity of the problem, which is the reason for us to call the resulting algorithms optimal. In particular, we obtain the following tractability results. We use standard notions of tractability, see Section 3 for their definition.

Theorem 2. The problem of the uniform approximation of functions in $F_{r, M}^{d}$ with deterministic algorithms based on function values suffers from the curse of dimen- 
sionality, if and only if $M \in\left[2^{r} r !, \infty\right)$. If $M \in\left(r !, 2^{r} r !\right)$, it is quasi-polynomially tractable but not polynomially tractable. If $M \in(0, r !]$, it is polynomially tractable but not strongly polynomially tractable.

Note that the first two statements are also true for randomized algorithms.

Before we proceed to the proofs, let us introduce some further notation that is used in the paper. In the following, the term box refers to a product of $d$ nonempty subintervals $I_{j}$ of $[0,1]$, in formulas it takes the form $\prod_{j=1}^{d} I_{j}$. The dispersion of a finite subset $P$ of $[0,1]^{d}$ is the minimal number $\eta>0$ such that $P$ has non-empty intersection with every box of volume greater than $\eta$. For any $k \in \mathbb{N}$, the set of natural numbers up to $k$ is denoted by $[k]$. If $x_{i}$ is a real number for each $i$ in some finite set $J$, we set $x_{J}=\left(x_{i}\right)_{i \in J}$. We write $x_{J}=1$ if $x_{j}=1$ for all $j \in J$. If $I_{i}$ is an interval for each $i \in J$, then $I_{J}$ denotes the Cartesian product of these intervals. If we are given functions $f_{i}: I_{i} \rightarrow \mathbb{R}$ for each $i \in J$, their tensor product is denoted by $f_{J}: I_{J} \rightarrow \mathbb{R}$. Throughout the paper, $r$ and $d$ are natural numbers, $\varepsilon$ is an element of $(0,1)$ and $M$ is positive. The natural logarithm of a positive number $a$ is denoted by $\ln a$, its logarithm in base two by $\log _{2} a$.

\section{Algorithms}

We start with the observation that the construction of an $\varepsilon$-detector is sufficient to achieve the worst case error $\varepsilon$ with the algorithm $A_{P, m}$. Recall that a point set $P$ in $[0,1]^{d}$ is called an $\varepsilon$-detector for $F_{r, M}^{d}$, if it contains a non-zero of any function $f \in F_{r, M}^{d}$ with $\|f\|_{\infty}>\varepsilon$. Note that any such function is of the following form:

$$
\begin{gathered}
f=\bigotimes_{i=1}^{d} f_{i}, \quad \text { where } \quad f_{i}:[0,1] \rightarrow[-1,1] \quad \text { with }\left\|f_{i}^{(r)}\right\|_{\infty} \leq M \\
\text { and }\|f\|_{\infty}=\prod_{i=1}^{d}\left\|f_{i}\right\|_{\infty}>\varepsilon .
\end{gathered}
$$

Lemma 1. Let $r \in \mathbb{N}, d \in \mathbb{N}$ and $M>0$. If $P$ is an $\varepsilon$-detector for $F_{r, M}^{d}$ and $m$ is chosen as in (2), then Algorithm 1 satisfies

$$
\mathrm{e}\left(A_{P, m}\right) \leq \varepsilon \quad \text { and } \quad \operatorname{cost}\left(A_{P, m}\right) \leq|P|+m .
$$

Proof. Let $f \in F_{r, M}^{d}$. If $P$ contains a non-zero of $f$, Algorithm 1 returns an $\varepsilon$-approximation of $f$ due to relation (1). If not, the output is zero. But since $P$ 
is a detector, we necessarily have $\|f\|_{\infty} \leq \varepsilon$ and zero is an $\varepsilon$-approximation of $f$, as well. The second statement is obvious.

Furthermore, we will use the following formula for polynomial interpolation.

Lemma 2. Let $a<b, r \in \mathbb{N}$ and $g \in W_{\infty}^{r}([a, b])$. Let $x_{1}, \ldots, x_{r} \in[a, b]$ be distinct and $p$ be the unique polynomial with degree less than $r$ such that $p\left(x_{i}\right)=g\left(x_{i}\right)$ for all $i \in[r]$. For every $x \in[a, b]$, there exist $\xi_{1}, \xi_{2} \in[a, b]$ such that

$$
g(x)-p(x)=\frac{1}{r !} \cdot \frac{g^{(r-1)}\left(\xi_{2}\right)-g^{(r-1)}\left(\xi_{1}\right)}{\xi_{2}-\xi_{1}} \cdot \prod_{i=1}^{r}\left(x-x_{i}\right)
$$

Lemma 2 is well known for $g \in \mathcal{C}^{r}([a, b])$. In this case, the second fraction can be replaced by $g^{(r)}(\xi)$ for some $\xi \in[a, b]$. We refer to [3, Theorem 2, Section 6.1]. Under the more general assumption that $g \in W_{\infty}^{r}([a, b])$, we have to modify the proof of the mentioned theorem.

Proof. If $x$ coincides with one of the nodes, the statement is trivial. Hence, let $x$ be distinct from all the nodes. We consider

$$
w:[a, b] \rightarrow \mathbb{R}, \quad w(y)=\prod_{i=1}^{r}\left(y-x_{i}\right)
$$

and set

$$
\lambda=\frac{g(x)-p(x)}{w(x)} .
$$

The function $\phi=g-p-\lambda w$ vanishes at the points $x_{1}, \ldots, x_{r}$ and $x$. Since $g$ and $\phi$ are elements of $W_{\infty}^{r}([a, b])$, their $(r-1)^{\text {st }}$ derivatives are absolutely continuous. If we apply Rolle's Theorem $(r-1)$ times, we obtain that $\phi^{(r-1)}$ has at least 2 distinct zeros $\xi_{1}$ and $\xi_{2}$ in $[a, b]$ and hence

$0=\int_{\xi_{1}}^{\xi_{2}} \phi^{(r)}(y) \mathrm{d} y=\int_{\xi_{1}}^{\xi_{2}} g^{(r)}(y)-\lambda r ! \mathrm{d} y=g^{(r-1)}\left(\xi_{2}\right)-g^{(r-1)}\left(\xi_{1}\right)-\lambda r !\left(\xi_{2}-\xi_{1}\right)$

This is the stated identity in disguise.

If $g \in W_{\infty}^{r}([0,1])$ has $r$ distinct zeros $x_{1}, \ldots, x_{r} \in[0,1]$, and $x$ is a maximum point of $|g|$, we get

$$
\|g\|_{\infty} \leq \frac{\left\|g^{(r)}\right\|_{\infty}}{r !} \prod_{i=1}^{r}\left|x-x_{i}\right|
$$


This follows from Lemma 2 since the unique polynomial $p$ with degree less than $r$ and $p\left(x_{i}\right)=g\left(x_{i}\right)$ for $i \in[r]$ is the zero polynomial and

$$
\left|g^{(r-1)}\left(\xi_{2}\right)-g^{(r-1)}\left(\xi_{1}\right)\right|=\left|\int_{\xi_{1}}^{\xi_{2}} g^{(r)}(y) \mathrm{d} y\right| \leq\left\|g^{(r)}\right\|_{\infty} \cdot\left|\xi_{2}-\xi_{1}\right| .
$$

The rest of this section is devoted to the construction of small $\varepsilon$-detectors for $F_{r, M}^{d}$. Thanks to Lemma 1 , this is sufficient to prove Theorem 1 . We will use three different strategies for three different ranges of the parameter $M$.

\subsection{Detectors for large derivatives}

In this section, the smoothness parameter $M$ can be arbitrarily large. It is shown in [8] that the cost of any algorithm with worst case error smaller than 1 is at least $2^{d}$ if $M \geq 2^{r} r$ !. In particular, the cardinality of any $\varepsilon$-detector must grow exponentially with the dimension. Yet, it does not get any worse: We construct an $\varepsilon$-detector whose cardinality "only" grows exponentially with the dimension but not super-exponentially. We use the following lemma.

Lemma 3. For each $g \in W_{\infty}^{r}([0,1])$ with $\left\|g^{(r)}\right\|_{\infty} \leq M$ there is a subinterval of $[0,1]$ with length

$$
L(g)=\min \left\{\frac{1}{r},\left(\frac{\|g\|_{\infty}}{M}\right)^{1 / r}\right\}
$$

which does not contain any zero of $g$.

Proof. The function $|g|$ attains its maximum, say for $x \in[0,1]$. We choose an interval $I \subset[0,1]$ of length $r L(g)$ that contains $x$. There are $r$ open and disjoint subintervals of $I$ with length $L(g)$. We label these intervals $I_{1}, \ldots, I_{r}$ such that the distance of $x$ and $I_{i}$ is increasing with $i$. Assume that every interval $I_{i}$ contains a zero $x_{i}$ of $g$. Then we have $\left|x-x_{i}\right|<i L(g)$ for all $i \in[r]$ and (5) leads to

$$
\|g\|_{\infty} \leq \frac{M}{r !} \prod_{i=1}^{r}\left|x-x_{i}\right|<M L(g)^{r} \leq\|g\|_{\infty} .
$$

This is a contradiction and the assertion is proven.

If, in addition, the uniform norm of $g$ is bounded by 1 , we have

$$
L(g) \geq \varrho^{-1}\|g\|_{\infty}^{1 / r} \quad \text { for } \quad \varrho=\max \left\{r, M^{1 / r}\right\}
$$


Hence, for every $f$ satisfying (4) there is a box $B$ in $[0,1]^{d}$ with volume

$$
\prod_{i \in[d]} L\left(f_{i}\right) \geq \varrho^{-d} \prod_{i \in[d]}\left\|f_{i}\right\|_{\infty}^{1 / r}=\varrho^{-d}\|f\|_{\infty}^{1 / r}>\varrho^{-d} \varepsilon^{1 / r}
$$

such that $f$ does not vanish anywhere on $B$. Hence, any point set $P$ in $[0,1]^{d}$ with dispersion $\varrho^{-d} \varepsilon^{1 / r}$ or less is an $\varepsilon$-detector for $F_{r, M}^{d}$. We know from the estimate of Larcher, see [1], that we can choose $P$ as a $(t, s, d)$-net with cardinality

$$
|P|=\left\lceil 2^{7 d+1} \varrho^{d} \varepsilon^{-1 / r}\right\rceil .
$$

By Lemma 1, the resulting algorithm achieves the worst case error $\varepsilon$ with the cost

$$
\operatorname{cost}\left(A_{P, m}\right) \leq\left\lceil 2^{7 d+1} \varrho^{d} \varepsilon^{-1 / r}\right\rceil+C_{r, M} d^{1+1 / r} \varepsilon^{-1 / r}
$$

This proves the first statement of Theorem 1 with $c_{1}=2^{8} \varrho+C_{r, M}$. Note that the cost of this algorithm has the minimal order of growth with respect to $\varepsilon$. It grows like $\varepsilon^{-1 / r}$ if $d$ is fixed and $\varepsilon$ tends to zero.

\subsection{Detectors for moderately large derivatives}

In this section, we assume that $M<2^{r} r$ !. In this case, we construct detectors $P$ with a cardinality that only grows polynomially with $d$ for any fixed $\varepsilon$. The construction of $P$ is based on the observation that for any function $f$ from (4) only some of the factors $f_{i}$ can have more than $(r-1)$ zeros close to $1 / 2$. This is an essential difference to the case $M \in\left[2^{r} r !, \infty\right)$, where all factors $f_{i}$ may have infinitely many zeros in any neighborhood of $1 / 2$. We are going to specify this statement in Lemma 5 , but first we need the following observation. For $\delta \in(0,1 / 2]$, we consider the interval $I_{\delta}:=[1 / 2-\delta, 1 / 2+\delta]$.

Lemma 4. Let $g \in W_{\infty}^{r}([0,1])$ with $\left\|g^{(r)}\right\|_{\infty} \leq M$. Assume that $g$ has $r$ distinct zeros in $I_{\delta}$. Then

$$
\|g\|_{\infty} \leq C_{\delta}:=\frac{M(1+2 \delta)^{r}}{2^{r} r !} .
$$

Proof. Let $x_{1}, \ldots, x_{r}$ be those zeros. The function $|g|$ attains its maximum, say for $x \in[0,1]$. By $(5)$ we have

$$
\|g\|_{\infty} \leq \frac{\left\|g^{(r)}\right\|_{\infty}}{r !} \prod_{i=1}^{r}\left|x-x_{i}\right| .
$$


This yields the desired inequality since $\left|x-x_{i}\right| \leq 1 / 2+\delta$ for each $i \in[r]$.

Since $M<2^{r} r$ !, we can choose $\delta \in(0,1 / 2]$ such that $C_{\delta}<1$. We define the pseudo-dimension $d_{0}$ as the largest number in $[d] \cup\{0\}$ that satisfies $C_{\delta}^{d_{0}}>\varepsilon$, that is

$$
d_{0}:=\min \left\{\left\lceil\frac{\ln \varepsilon}{\ln C_{\delta}}\right\rceil-1, d\right\} .
$$

Obviously, the pseudo-dimension is bounded above independently of $d$. We can now specify the statement from the beginning of this section.

Lemma 5. Let $f$ be given as in (4). Then there are at most $d_{0}$ coordinates $i \in[d]$ such that $f_{i}$ has more than $(r-1)$ zeros in $I_{\delta}$.

Proof. Let $k$ be the number of coordinates $i \in[d]$ for which $f_{i}$ has more than $(r-1)$ zeros in $I_{\delta}$. Lemma 4 yields that $\varepsilon<\|f\|_{\infty} \leq C_{\delta}^{k}$. The maximality of $d_{0}$ yields that $k \leq d_{0}$.

This means that there is a subset $J^{*}$ of $[d]$ with cardinality $d_{0}$ such that $f_{i}$ has at most $(r-1)$ zeros in $I_{\delta}$ for all $i \in[d] \backslash J^{*}$. We can find a non-zero of $f$, if we solve the following tasks:

\section{Find $J^{*}$. 2. Find a non-zero of $f_{J^{*}}$ 3. Find a non-zero of $f_{[d] \backslash J^{*}}$.}

Let us go through these tasks one by one. We will deal with the first task by simply going through all possible sets $J \subset[d]$ of cardinality $d_{0}$. The number of such sets only depends polynomially on $d$. We can cope with the second task, since this problem is only $d_{0}$-dimensional. By Lemma 3 , there is a box $B$ in $[0,1]^{d_{0}}$ with volume

$$
\prod_{i \in J^{*}} L\left(f_{i}\right) \geq \prod_{i \in J^{*}} \varrho^{-1}\left\|f_{i}\right\|_{\infty}^{1 / r} \geq \varrho^{-d_{0}} \prod_{i \in[d]}\left\|f_{i}\right\|_{\infty}^{1 / r}=\varrho^{-d_{0}}\|f\|_{\infty}^{1 / r}>\varrho^{-d_{0}} \varepsilon^{1 / r}
$$

such that $f_{J^{*}}$ does not vanish on $B$. Hence, any point set $P_{1}$ in $[0,1]^{d_{0}}$ with dispersion $\varrho^{-d_{0}} \varepsilon^{1 / r}$ or less contains a non-zero of $f_{J^{*}}$. Again by the result of Larcher, see [1], we know that we can choose $P_{1}$ as a $(t, s, d)$-net of cardinality $2^{7 d_{0}+1} \varrho^{d_{0}} \varepsilon^{-1 / r}$. The third task is also feasible, since $f_{i}$ has at most $(r-1)$ zeros in $I_{\delta}$ for all $i \in[d] \backslash J^{*}$. We use the following observation.

Lemma 6. Let $J$ be an $\ell$-element subset of $[d]$ and, for every $i \in J$, let $f_{i}$ be a function with at most $k$ zeros on some interval $I_{i}$. Then every $(\ell k+1)$-element set in $I_{J}$ whose elements are pairwise distinct in every coordinate contains a non-zero of $f_{J}$. 
Proof. Let $P$ be an $(\ell k+1)$-element set in $I_{J}$ whose elements are pairwise distinct in every coordinate and suppose that $f_{J}$ vanishes everywhere on $P$. For each $i \in J$ let $P_{i}=\left\{x_{J} \in P \mid f_{i}\left(x_{i}\right)=0\right\}$. Since $f_{J}\left(x_{J}\right)=0$ implies that there is some $i \in J$ with $f_{i}\left(x_{i}\right)=0$, we have $P=\bigcup_{i \in J} P_{i}$. This can only be true, if one of the sets $P_{i}$ has more than $k$ elements. But since $x_{i}$ is different for every $x_{J} \in P_{i}$, this means that the corresponding function $f_{i}$ has more than $k$ zeros, a contradiction.

We apply this lemma for the functions $f_{i}$ in (4), for the index set $J=[d] \backslash J^{*}$ and for $k=r-1$. We obtain that the diagonal set

$$
P_{2}=\left\{\left(\frac{1}{2}-\delta+\frac{2 \delta j}{(r-1)\left(d-d_{0}\right)}\right) \cdot \mathbf{1} \mid j \in \mathbb{N}_{0} \text { with } j \leq(r-1)\left(d-d_{0}\right)\right\}
$$

in $[0,1]^{d-d_{0}}$ contains a non-zero of $f_{[d] \backslash J^{*}}$. All together, we obtain the detector

$$
P=\bigcup_{J \subset[d]:|J|=d_{0}}\left\{x \in[0,1]^{d} \mid x_{J} \in P_{1}, x_{[d] \backslash J} \in P_{2}\right\}
$$

In fact, we have seen above that for any $f$ satisfying (4) there must be some $J^{*} \subset[d]$ with $\left|J^{*}\right|=d_{0}$, a non-zero $y \in P_{1}$ of $f_{J^{*}}$ and a non-zero $z \in P_{2}$ of $f_{[d] \backslash J^{*}}$. The point $x \in[0,1]^{d}$ with $x_{J^{*}}=y$ and $x_{[d] \backslash J^{*}}=z$ is contained in the set $P$ and a non-zero of $f$. The cardinality of the detector is given by

$$
|P|=\left(\begin{array}{c}
d \\
d_{0}
\end{array}\right)\left|P_{1}\right|\left|P_{2}\right|=\left(\begin{array}{c}
d \\
d_{0}
\end{array}\right)\left[(r-1)\left(d-d_{0}\right)+1\right] 2^{7 d_{0}+1} \varrho^{d_{0}} \varepsilon^{-1 / r} .
$$

This number grows like $d^{d_{0}+1}$ if $\varepsilon$ is fixed and $d$ tends to infinity. Together with Lemma 1, this proves the second statement of Theorem 1 with

$$
c_{2}=2 r+C_{r, M}, \quad \text { and } \quad c_{3}=\ln \left(2^{7} \varrho\right)\left(1+1 / \ln \left(C_{\delta}^{-1}\right)\right) .
$$

Note that $d_{0}$ equals $d$ if $\varepsilon$ is small enough. Hence, the cardinality of $P$ and the cost of the algorithm grows like $\varepsilon^{-1 / r}$ if $d$ is fixed and $\varepsilon$ tends to zero, which is optimal.

\subsection{Detectors for small derivatives}

In this section, we assume that $M \leq r$ !. In this case, each function $f$ satisfying (4) does not vanish almost everywhere on a box whose size is independent of $d$. This is due to the following fact. 
Lemma 7. For each $g \in W_{\infty}^{r}([0,1])$ with $\|g\|_{\infty} \leq 1$ and $\left\|g^{(r)}\right\|_{\infty} \leq r$ ! there is an interval in $[0,1]$ with length $\|g\|_{\infty}^{1 / r}$ which contains at most $(r-1)$ zeros of $g$.

Proof. The function $|g|$ attains its maximum, say for $x \in[0,1]$. We choose an open interval $I \subset[0,1]$ of length $\|g\|_{\infty}^{1 / r}$ whose closure contains $x$. Assume that $I$ contains $r$ distinct zeros $x_{1}, \ldots, x_{r}$ of $g$. Then $\left|x-x_{i}\right|<\|g\|_{\infty}^{1 / r}$ for all $i \in[r]$ and (5) yields

$$
\|g\|_{\infty} \leq \frac{\left\|g^{(r)}\right\|_{\infty}}{r !} \prod_{j=1}^{r}\left|x-x_{j}\right| \leq \prod_{j=1}^{r}\left|x-x_{j}\right|<\|g\|_{\infty} .
$$

This is a contradiction and the assertion is proven.

We now construct an $\varepsilon$-detector for any $\varepsilon \in(0,1)$. To this end, let

$$
\gamma=\left(1-2^{-1 / d}\right) \varepsilon^{1 / r}
$$

Note that $\gamma$ is smaller than $1 / 2$. We choose a point set $P_{0}$ in $[0,1]^{d}$ whose dispersion is at most $\varepsilon^{1 / r} / 2$ and consider the point set $P$ in $[0,1]^{d}$, given by

$$
P=\left\{(1-\gamma) \cdot x+\frac{\gamma j}{(r-1) d} \cdot \mathbf{1} \mid x \in P_{0} \text { and } j \in \mathbb{N}_{0} \text { with } j \leq(r-1) d\right\} .
$$

Lemma 8. The point set $P$ is an $\varepsilon$-detector for $F_{r, M}^{d}$.

Proof. Let $f$ be given as in (4). By Lemma 7 , there are intervals $\left(a_{i}, b_{i}\right)$ in $[0,1]$ with length $\left\|f_{i}\right\|_{\infty}^{1 / r}$ containing at most $(r-1)$ zeros of $f_{i}$. By the choice of $\gamma$, we have

$$
\gamma \leq\left(1-2^{-1 / d}\right)\left\|f_{i}\right\|_{\infty}^{1 / r}
$$

In particular, the box

$$
\widetilde{B}=\prod_{i \in[d]}\left(a_{i}, b_{i}-\gamma\right)
$$

is well defined. In fact, the volume of this box satisfies

$$
|\widetilde{B}|=\prod_{i \in[d]}\left(\left\|f_{i}\right\|_{\infty}^{1 / r}-\gamma\right) \geq \prod_{i \in[d]}\left(2^{-1 / d}\left\|f_{i}\right\|_{\infty}^{1 / r}\right)=\frac{\|f\|_{\infty}^{1 / r}}{2}>\frac{\varepsilon^{1 / r}}{2}
$$

The box $\widetilde{B} /(1-\gamma)$ is contained in $[0,1]^{d}$ and even larger than $\widetilde{B}$. It hence contains some $x \in P_{0}$. Consequently, we have $(1-\gamma) x \in \widetilde{B}$ and all the points

$$
x^{(j)}=(1-\gamma) \cdot x+\frac{\gamma j}{(r-1) d} \cdot \mathbf{1} \quad \text { for } j \in \mathbb{N}_{0} \text { with } j \leq(r-1) d
$$


are elements of $P$. These are $(r-1) d+1$ points that are pairwise distinct in every coordinate and that are all contained in the larger box

$$
B=\prod_{i \in[d]}\left(a_{i}, b_{i}\right)
$$

Recall that each function $f_{i}$ has at most $(r-1)$ zeros in $\left(a_{i}, b_{i}\right)$. By Lemma 6 , one of the points $x^{(j)}$ must be a non-zero of $f$. As an example, Figure 2.3 illustrates the case $d=2$ and $r=3$.

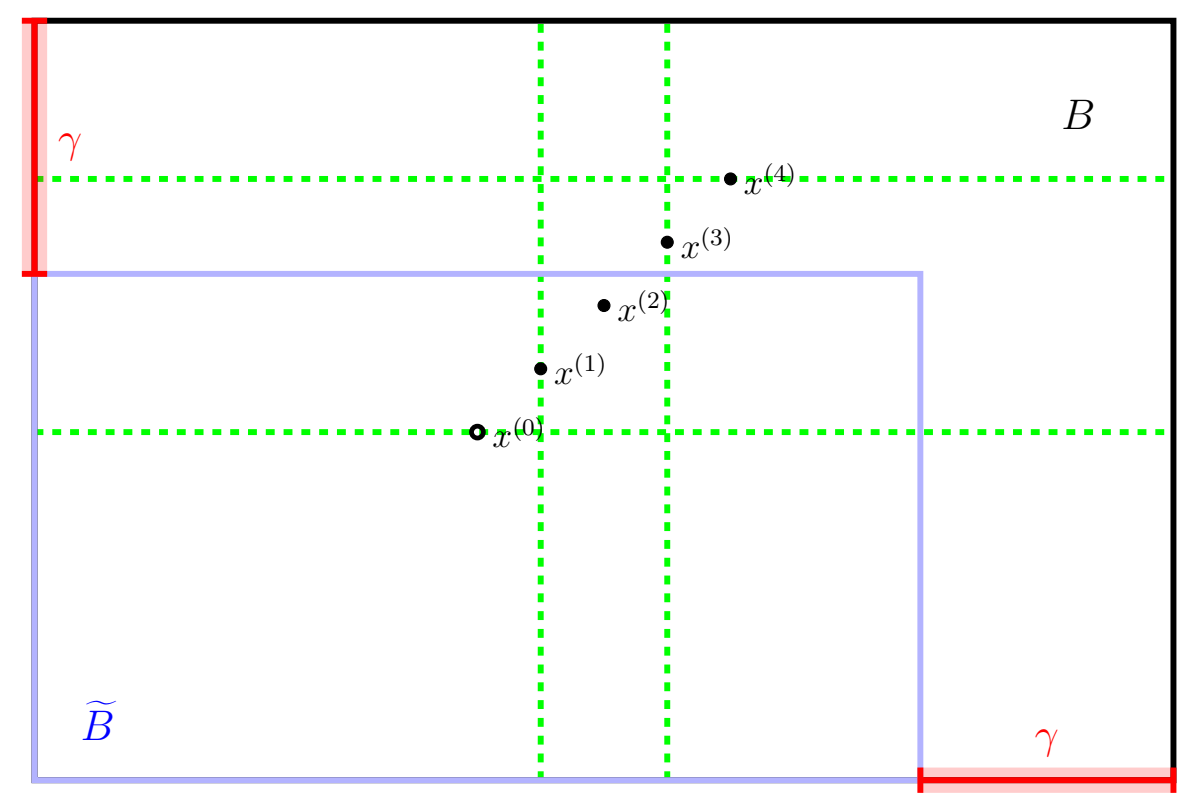

Figure 1: The box $B$ for $(r, d)=(3,2)$. The dashed lines indicate the zeros of $f$ in $B$. Since $f$ only vanishes there, one of the points $x^{(j)}$ must be a non-zero.

This means that we have an $\varepsilon$-detector for $F_{r, M}^{d}$ with the cardinality

$$
|P|=((r-1) d+1)\left|P_{0}\right|
$$

where $P_{0}$ is a point set with dispersion $\varepsilon^{1 / r} / 2$ or less. For example, we know from [9] that this can be achieved with

$$
\left|P_{0}\right|=\left\lceil 2^{4} d \varepsilon^{-1 / r} \ln \left(66 \varepsilon^{-1 / r}\right)\right\rceil
$$

points. In particular, Lemma 8 and Lemma 1 give the last statement of Theorem 1 with the constant $c_{4}=85 r+C_{r, M}$. 
Remark 1. Based on [10], it has recently been shown in [11] that $P_{0}$ can also be chosen such that

$$
\left|P_{0}\right|=\left\lceil 2^{11} \log d \log ^{2}\left(2 \varepsilon^{-1 / r}\right) \varepsilon^{-2 / r}\right\rceil .
$$

This number is smaller than (6) if $d$ is large, but the dependence on $\varepsilon$ is worse.

Remark 2. In contrast to the algorithms for large and moderate derivatives, the algorithm for small derivatives is not completely explicit since we do not know how to construct such point sets $P_{0}$. We only know that they exist. So far, the only construction of a point set that achieves the desired dispersion and only grows polynomially with the dimension is given in [4]. However, the order of growth is proportional to $\log \left(\varepsilon^{-1}\right)$ and the resulting algorithm would not improve on the algorithm for moderate derivatives.

\section{Lower bounds}

In this section we provide lower bounds on the complexity of the uniform approximation problem on $F_{r, M}^{d}$. Together with the upper bounds from Section 2, this proves the tractability results of Theorem 2 . First, we recall the relevant notions of tractability. For every $n \in \mathbb{N}$, the $n$th minimal worst case error is given by

$$
e(n, d):=\inf _{A_{n}} e\left(A_{n}\right)
$$

where the infimum is taken over all adaptive algorithms $A_{n}$ that use at most $n$ function values. The algorithms are of the form $A_{n}(f)=\phi\left(f\left(x_{1}\right), \ldots, f\left(x_{n}\right)\right)$ with $\phi: \mathbb{R}^{n} \rightarrow L_{\infty}$, where the $x_{i} \in[0,1]^{d}$ can be chosen adaptively, depending on the already known function values $f\left(x_{1}\right), \ldots, f\left(x_{i-1}\right)$, see for example [6, 7]. We also need the inverse of the minimal worst case error

$$
n(\varepsilon, d):=\inf \{n \mid e(n, d) \leq \varepsilon\}
$$

We say that the uniform recovery problem on $F_{r, M}^{d}$

- is strongly polynomially tractable if there are $c, p>0$ such that $n(\varepsilon, d) \leq$ $c \varepsilon^{-p}$ for all $\varepsilon \in(0,1)$ and all $d \in \mathbb{N}$;

- is polynomially tractable if there are $c, q, p>0$ such that $n(\varepsilon, d) \leq c \varepsilon^{-p} d^{q}$ for all $\varepsilon \in(0,1)$ and all $d \in \mathbb{N}$; 
- is quasi-polynomially tractable if there are $c, t>0$ such that

$$
n(\varepsilon, d) \leq c \exp \left(t\left(1+\ln \left(\varepsilon^{-1}\right)\right)(1+\ln d)\right)
$$

for all $\varepsilon \in(0,1)$ and all $d \in \mathbb{N}$;

- suffers from the curse of dimensionality if there is some $\varepsilon>0, c>0$ and $\alpha>1$ such that $n(\varepsilon, d) \geq c \alpha^{d}$.

Note that the results of Section 2 imply that the problem is quasi-polynomially tractable if $M<2^{r} r$ ! and polynomially tractable if $M \leq r$ !. We now provide the respective lower bounds.

The case $M \in\left[2^{r} r !, \infty\right)$. Here, the problem suffers from the curse of dimensionality. This has already been shown in [8, Theorem 2]. For the reader's convenience, we repeat the proof. The function

$$
g(x)=2^{r}(x-1 / 2)^{r} \cdot \mathbf{1}_{[0,1 / 2]}(x), \quad x \in[0,1]
$$

is $r$-times differentiable with $\|g\|_{\infty}=1$ and $\left\|g^{(r)}\right\|_{\infty} \leq M$. The same holds for the function

$$
h(x)=2^{r}(x-1 / 2)^{r} \cdot \mathbf{1}_{[1 / 2,1]}(x), \quad x \in[0,1] .
$$

Hence, all functions $f=f_{[d]}$ with $f_{i} \in\{g, h\}$ for $i \in[d]$ are contained in $F_{r, M}^{d}$ and satisfy $\|f\|_{\infty}=1$. These are $2^{d}$ functions with pairwise disjoint support.

Let $A$ be an algorithm and let $x_{1}, \ldots, x_{n}$ be the sample points the algorithm uses for the input $f_{0}=0$. If $n<2^{d}$, there is at least one $f$ from the $2^{d}$ functions defined above that vanishes at all these points. Therefore, the algorithm cannot distinguish $f$ and $-f$ from $f_{0}$ such that

$$
A(f)=A\left(f_{0}\right)=A(-f)
$$

and we obtain the error bound

$$
\mathrm{e}(A) \geq \max \left\{\left\|A\left(f_{0}\right)-f\right\|_{\infty},\left\|A\left(f_{0}\right)+f\right\|_{\infty}\right\} \geq\|f\|_{\infty}=1 .
$$

Hence, the problem suffers from the curse of dimensionality: For any $\varepsilon \in(0,1)$ we have that $n(\varepsilon, d) \geq 2^{d}$. 
The case $M \in\left(r !, 2^{r} r !\right)$. Note that the point $x_{0}=(r ! / M)^{1 / r}$ is contained in $(1 / 2,1)$. The function

$$
g(x)=\frac{M\left(x-x_{0}\right)^{r}}{r !} \cdot \mathbf{1}_{\left[0, x_{0}\right]}(x), \quad x \in[0,1]
$$

is $r$-times differentiable with $\|g\|_{\infty}=1$ and $\left\|g^{(r)}\right\|_{\infty} \leq M$. The function

$$
h(x)=\frac{M\left(x-x_{0}\right)^{r}}{r !} \cdot \mathbf{1}_{\left[x_{0}, 1\right]}(x), \quad x \in[0,1]
$$

is also $r$-times differentiable with $\left\|h^{(r)}\right\|_{\infty} \leq M$ and $\|h\|_{\infty}=|h(1)|$ is in $(0,1)$. Let $k(\varepsilon, d)$ be the largest number in $[d] \cup\{0\}$ such that $|h(1)|^{k(\varepsilon, d)}>\varepsilon$. Namely, let

$$
k(\varepsilon, d)=\min \{\kappa(\varepsilon), d\} \quad \text { with } \quad \kappa(\varepsilon):=\left\lceil\frac{\ln \left(\varepsilon^{-1}\right)}{\ln \left(|h(1)|^{-1}\right)}\right\rceil-1 .
$$

For every subset $J$ of $[d]$ with cardinality $k(\varepsilon, d)$, the function $f=f_{[d]}$ with $f_{i}=g$ for $i \in J$ and $f_{i}=h$ for $i \in[d] \backslash J$ is contained in $F_{r, M}^{d}$ and satisfies $\|f\|_{\infty}>\varepsilon$. These are $\left(\begin{array}{c}d \\ k(\varepsilon, d)\end{array}\right)$ functions with pairwise disjoint support.

Let $A$ be an algorithm and let $x_{1}, \ldots, x_{n}$ be the sample points the algorithm uses for the input $f_{0}=0$. If $n<\left(\begin{array}{c}d \\ k(\varepsilon, d)\end{array}\right)$, there is at least one from the $\left(\begin{array}{c}d \\ k(\varepsilon, d)\end{array}\right)$ functions defined above that vanishes at all these points. Therefore, the algorithm cannot distinguish $f$ and $-f$ from $f_{0}$, such that its error satisfies

$$
\mathrm{e}(A) \geq \max \left\{\left\|A\left(f_{0}\right)-f\right\|_{\infty},\left\|A\left(f_{0}\right)+f\right\|_{\infty}\right\} \geq\|f\|_{\infty}>\varepsilon .
$$

We obtain

$$
n(\varepsilon, d) \geq\left(\begin{array}{c}
d \\
k(\varepsilon, d)
\end{array}\right) \geq\left(\frac{d}{k(\varepsilon, d)}\right)^{k(\varepsilon, d)} .
$$

This implies that the problem is not polynomially tractable: In fact, let us assume that the problem is polynomially tractable. Then there are $c, q, p>0$ such that

$$
n(\varepsilon, d) \leq c \varepsilon^{-p} d^{q}
$$

for all $\varepsilon \in(0,1)$ and all $d \in \mathbb{N}$. We can, however, choose $\varepsilon \in(0,1)$ such that $\kappa(\varepsilon)>q$ and hence

$$
\lim _{d \rightarrow \infty} \frac{n(\varepsilon, d)}{d^{q}} \geq \lim _{d \rightarrow \infty} \frac{d^{\kappa(\varepsilon)-q}}{\kappa(\varepsilon)^{\kappa(\varepsilon)}}=\infty
$$

which contradicts the assumption (7).

The case $M \in(0, r !]$. First, we consider the case $r \geq 2$. Let $A$ be an algorithm 
and let $x_{1}, \ldots, x_{n}$ be the sample points the algorithm uses for the input $f_{0}=0$. Let us assume that $n \leq d$. For each $i \in[n]$, there is a linear function $f_{i}$ on $[0,1]$ that vanishes at the $i$-th coordinate of $x_{i}$ and satisfies $\left\|f_{i}\right\|_{\infty}=1$. For $i \in[d] \backslash[n]$ we set $f_{i}=1$. The function $f=f_{[d]}$ is in $F_{r, M}^{d}$ and vanishes at all sample points. Hence, $f$ and $-f$ cannot be distinguished from $f_{0}$ and the error of $A$ is at least $\|f\|_{\infty}=1$. This implies that $n(\varepsilon, d)>d$ for any $\varepsilon<1$. In particular, the problem is not strongly polynomially tractable.

Now assume that $r=1$. The previous argument does not work in this case, since the first derivative of $f_{i}$ is not necessarily bounded by $M$. Here, we assume that the number of sample points of the algorithm $A$ for the input $f_{0}=0$ is at most $\left\lfloor\log _{2} d\right\rfloor$. By the proof of [1, Lemma 2], we know that there are two distinct coordinates $j, \ell \in[d]$ such that the box $I_{[d]}$ does not contain any of these points, where $I_{j}=[0,1 / 2), I_{\ell}=(1 / 2,1]$ and $I_{i}=[0,1]$ otherwise. The function $f=f_{[d]}$ with

$$
f_{i}(x)=M(x-1 / 2) \cdot \mathbf{1}_{I_{i}}(x), \quad x \in[0,1]
$$

for $i \in\{j, \ell\}$ and $f_{i}=1$ otherwise, is contained in $F_{r, M}^{d}$ and vanishes at all sample points. Therefore, the algorithm cannot distinguish $f$ and $-f$ from $f_{0}$ such that its error is at least $\|f\|_{\infty}=M^{2} / 4$. This implies that $n(\varepsilon, d)>\left\lfloor\log _{2} d\right\rfloor$ for any $\varepsilon<M^{2} / 4$. In particular, the problem is not strongly polynomially tractable.

Remark 3. In [8, Theorem 3] the curse of dimensionality for $M \in\left[2^{r} r !, \infty\right)$ is also proven for randomized algorithms. Similarly, one can also extend the lower bound for the case $M \in\left(r !, 2^{r} r\right.$ !) to randomized algorithms by using a technique of Bakhvalov, see [5, Section 2.2.2] for details.

Acknowledgements. Both authors were supported by the Erwin Schrödinger International Institute for Mathematical Physics during the program on Tractability of High Dimensional Problems and Discrepancy. Daniel Rudolf gratefully acknowledges support of the Felix-Bernstein-Institute for Mathematical Statistics in the Biosciences (Volkswagen Foundation) and the Campus laboratory AIMS.

\section{References}

[1] C. Aistleitner, A. Hinrichs and D. Rudolf: On the size of the largest empty box amidst a point set. Discrete Applied Mathematics 230, 146-150, 2017.

[2] M. Bachmayr, W. Dahmen, R. DeVore and L. Grasedyck: Approximation of high-dimensional rank one tensors. Constr. Approx. 39, 385-395, 2014. 
[3] D. Kincaid and E. Cheney: Numerical Analysis: Mathematics of Scientific Computing. Brooks/Cole, Pacific Grove, Calif., 1991.

[4] D. Krieg: On the dispersion of sparse grids. J. Complexity 45, 115-119, 2018.

[5] E. Novak: Deterministic and stochastic error bounds in numerical analysis. LNiM 1349, Springer-Verlag, Berlin, 1988.

[6] E. Novak: On the power of adaption. J. Complexity 12, 199-237, 1996.

[7] E. Novak and H. Woźniakowski: Tractability of multivariate problems. Volume I: Linear Information, European Math. Soc. Publ. House, Zürich, 2008 .

[8] E. Novak and D. Rudolf: Tractability of the approximation of high-dimensional rank one tensors. Constr. Approx. 43, 1-13, 2016.

[9] D. Rudolf: An upper bound on the minimal dispersion via delta covers. Contemporary Computational Mathematics - A Celebration of the 80th Birthday of Ian Sloan, Springer-Verlag, 1099-1108, 2018.

[10] J. Sosnovec: A note on the minimal dispersion of point sets in the Unit Cube. European J. Combinatorics 69, 255-259, 2018.

[11] M. Ullrich and J. Vybíral: An upper bound on the minimal dispersion. J. Complexity 45, 120-126, 2018. 